\title{
Article \\ Clinicopathological Significance of Syndecan-1 in Cholangiocarcinoma: A Study Based on Immunohistochemistry and Public Sequencing Data
}

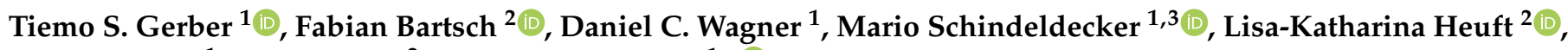 \\ Wilfried Roth ${ }^{1}$, Hauke Lang ${ }^{2}$ and Beate K. Straub ${ }^{1, *(1)}$ \\ 1 Institute of Pathology, University Medical Center of the Johannes Gutenberg-University Mainz, \\ 55131 Mainz, Germany; tiemo.gerber@unimedizin-mainz.de (T.S.G.); \\ Daniel-Christoph.Wagner@unimedizin-mainz.de (D.C.W.); \\ mario.schindeldecker@unimedizin-mainz.de (M.S.); wilfried.roth@unimedizin-mainz.de (W.R.) \\ 2 Department of General, Visceral and Transplant Surgery, University Medical Center of the Johannes \\ Gutenberg-University Mainz, 55131 Mainz, Germany; fabian.bartsch@unimedizin-mainz.de (F.B.); \\ lisa-katharina.heuft@unimedizin-mainz.de (L.-K.H.); Hauke.Lang@unimedizin-mainz.de (H.L.) \\ 3 Tissue Biobank, University Medical Center of the Johannes Gutenberg-University Mainz, \\ 55131 Mainz, Germany \\ * Correspondence: beate.straub@unimedizin-mainz.de
}

\section{check for} updates

Citation: Gerber, T.S.; Bartsch, F.; Wagner, D.C.; Schindeldecker, M.; Heuft, L.-K.; Roth, W.; Lang, H.; Straub, B.K. Clinicopathological Significance of Syndecan-1 in Cholangiocarcinoma: A Study Based on Immunohistochemistry and Public Sequencing Data. J. Clin. Med. 2021, 10, 2745. https://doi.org/10.3390/ jcm10132745

Academic Editor: Katsunori Yoshida

Received: 14 May 2021

Accepted: 19 June 2021

Published: 22 June 2021

Publisher's Note: MDPI stays neutral with regard to jurisdictional claims in published maps and institutional affiliations.

Copyright: (c) 2021 by the authors. Licensee MDPI, Basel, Switzerland. This article is an open access article distributed under the terms and conditions of the Creative Commons Attribution (CC BY) license (https:// creativecommons.org/licenses/by/ $4.0 /)$.

\begin{abstract}
Background: Syndecan-1 (CD138; SDC1) is a heparan sulfate proteoglycan that has been attributed a key role in cancer progression in ductal adenocarcinoma of the pancreas. We therefore aimed to investigate the role of syndecan- 1 in cholangiocarcinoma. Methods: We analyzed syndecan-1 expression in a large, clinicopathologically well-characterized collective of 154 intrahepatic cholangiocarcinoma, 221 extrahepatic cholangiocarcinomas, and 95 gallbladder carcinomas as well as respective normal tissues and precursor lesions by immunohistochemistry with digital image analysis and correlated with recurrence-free survival and prognostic markers. Furthermore, we conducted an analysis of cancer genes in the cholangiocarcinoma cohort of The Cancer Genome Atlas (TCGA). Results: During cholangiocarcinogenesis, syndecan-1-expression decreased when compared to normal bile ducts and biliary intraepithelial neoplasia; however, syndecan-1 levels were found to be elevated in lymph node metastases. In the TCGA cohort, high mRNA SDC1 levels were associated with poor prognosis in intrahepatic cholangiocarcinoma. However, in our large cohort, the immunohistochemical syndecan-1 expression did not significantly correlate with recurrence-free survival. Conclusions: Syndecan-1 was found to be downregulated during cholangiocarcinogenesis, yet we could not show significant effects on prognosis on protein level. Further analyses are needed to further depict its specific role.
\end{abstract}

Keywords: cholangiocarcinoma; syndecan-1; biomarker; SDC1

\section{Introduction}

Syndecans are a family of cell surface heparan proteoglycans. Syndecan-1 (CD138, encoded by the SDC1 gene [1]) is a transmembrane protein with an intra- and extracellular domain. It is expressed on the basolateral surface of epithelial cells, binds with its extracellular domain to extracellular matrix components, and associates with its intracellular domain to the actin cytoskeleton. Syndecan-1 plays a key role in the modulation of cancer cell proliferation and invasion, inflammation, and matrix remodeling. In the liver, syndecan- 1 is expressed on the sinusoidal and intercellular surface of hepatocytes and the basolateral surface of cholangiocytes of bile ducts, irrespective of size. Immunohistochemical expression is increased in specimens with liver cirrhosis and chronic cholestatic disease [2-4]. 
CD138 immunohistochemistry is currently widely used in routine histopathology to highlight plasma cells in different immunologic diseases as well as for the diagnosis of plasma cell myeloma. Anti-CD138 drugs are currently being evaluated in clinical trials for this disease [5].

Syndecan-1 was recently uncovered to be upregulated at the cell surface by $K R A S$ in pancreatic ductal adenocarcinoma (PDAC). KRAS mutations are the most common and earliest alteration in cancer development in PDAC, which is present in more than $90 \%$ of cases. Syndecan-1 is supposed to mediate macropinocytosis at the cell surface, which is involved in cancer growth and progression. In addition, macropinocytosis is associated with necrocytosis, a drug resistance mechanism for standard chemotherapeutic therapies, such as gemcitabine, 5-fluorouracil, and doxorubicin [6,7]. KRAS mutations, macrocytosis, and drug resistance are closely related factors [8-10]. Therefore, these findings are attracting considerable interest to biomarkers with a prognostic relevant association to macropinocytosis. For syndecan-1, a mechanistic link to KRAS signaling and macropinocytosis has recently been uncovered. This critical role of SDC1 has led to the suggestion of therapeutic interventions with antibody targeting $[7,11]$.

PDAC and carcinomas of the biliary tract stem from embryonically related cell types, and therefore might share similarities in tumor biology. Cholangiocarcinomas are a rare and heterogeneous group of malignant tumors with globally rising incidence. It is the second most common primary hepatic malignancy behind hepatocellular carcinoma. In the past decade, the prognosis of cholangiocarcinoma patients has not improved substantially [11]. Intrahepatic cholangiocarcinomas are aggressive malignancies with poor overall survival and a high probability of recurrence, even with negative tumor margin resection [12]. We aimed to elaborate whether syndecan-1 expression correlates to survival in cholangiocarcinoma and thereby parallels its function in PDAC.

\section{Materials and Methods}

Paraffin-embedded tissue samples from 470 patients with cholangiocarcinoma were provided by and in accordance with the regulations of the Tissue Biobank of the University Medical Center Mainz after approval by the local ethics committee of Rhineland-Palatinate. In our series, we included intra- and extrahepatic cholangiocarcinoma (iCCA and eCCA) as well as gallbladder carcinoma (GBC, $n=95)$, diagnosed at the Institute of Pathology, University Medical Center Mainz, between the years 2006 and 2020. Tissue samples were obtained from surgical specimens, and larger tissue samples were obtained from irresectable cases. Intrahepatic cholangiocarcinoma $(n=154)$ were reviewed and classified into small and large duct types according to the WHO classification of tumors of the digestive system (fifth edition, 2019). Depending on its location, ECCA were subdivided into perihilar (PHCC, $n=162$ ) and distal (dCCA, $n=59$ ) cholangiocarcinoma. PHCC were defined as primarily located in the hilus region in the common hepatic duct and possibly extending to the right or left hepatic duct with periductal growth. Small duct-type iCCA were defined as typically peripherally localized carcinomas with small tubular growth, low columnar tumor cells, without mucin secretion, and typically without perineural invasion, whereas iCCA, large duct type, were by definition typically centrally/periductally located carcinomas with the formation of large ducts and mucin production [13]. In addition, we also included biliary intraepithelial neoplasia (BilIn) and normal tissue (small, large and perihilar bile ducts, and gallbladder epithelium). Clinical follow-up and survival data were available in most cases (Table 1). Tissue microarray (TMA) blocks were generated with primary cancer, corresponding normal tissue, precursor lesions, and metastases. Subsequently, TMA images were digitalized by a whole slide scanner at $400 \times$, with a pixel size of $0.2278 \times 0.2278 \mu \mathrm{m}$ (Nanozoomer, Hamamatsu Photonics, Hamamatsu, Japan) and further analyzed. 
Table 1. Patient characteristics.

\begin{tabular}{|c|c|c|c|c|}
\hline & $\begin{array}{c}\text { iCCA } \\
n=154\end{array}$ & $\begin{array}{l}\mathrm{dCCA} \\
n=59\end{array}$ & $\begin{array}{c}\text { РНCC } \\
n=162\end{array}$ & $\begin{array}{c}\text { GBC } \\
n=95\end{array}$ \\
\hline Age $^{\dagger}$ & $64.02 \pm 10.78$ & $69.04 \pm 9.01$ & $66.94 \pm 11.01$ & $67.17 \pm 11.92$ \\
\hline Male & $59 \%$ & $71 \%$ & $62 \%$ & $35 \%$ \\
\hline Female & $41 \%$ & $29 \%$ & $38 \%$ & $65 \%$ \\
\hline \multicolumn{5}{|l|}{ Syndecan-1 } \\
\hline Intensity $^{+}$ & $1.72 \pm 0.77$ & $1.30 \pm 0.89$ & $1.46 \pm 0.82$ & $1.17 \pm 0.84$ \\
\hline H-score ${ }^{+}$ & $47.5 \pm 48.22$ & $38.28 \pm 44.59$ & $44.16 \pm 42.64$ & $39.67 \pm 47.67$ \\
\hline Proliferation $\ddagger$ & $17.75 \pm 15.83$ & $21.91 \pm 16.46$ & $18.39 \pm 15.92$ & $23.94 \pm 17.09$ \\
\hline pTX & 15 & 0 & 12 & 11 \\
\hline pT1 & 75 & 7 & 8 & 3 \\
\hline pT2 & 41 & 26 & 122 & 30 \\
\hline pT3 & 18 & 25 & 18 & 47 \\
\hline pT4 & 5 & 1 & 2 & 4 \\
\hline $\mathrm{pNX}$ & 51 & 0 & 32 & 31 \\
\hline pN0 & 70 & 31 & 87 & 31 \\
\hline $\mathrm{pN} 1 / 2$ & 33 & 28 & 43 & 33 \\
\hline G1 & 5 & 1 & 5 & 3 \\
\hline G2 & 117 & 37 & 104 & 43 \\
\hline G3 & 32 & 19 & 53 & 47 \\
\hline G4 & 0 & 2 & 0 & 2 \\
\hline L0 & 135 & 42 & 141 & 65 \\
\hline L1 & 19 & 17 & 21 & 30 \\
\hline V0 & 128 & 50 & 145 & 81 \\
\hline V1 & 26 & 9 & 17 & 14 \\
\hline Pn0 & 125 & 16 & 54 & 55 \\
\hline Pn1 & 29 & 43 & 108 & 40 \\
\hline R0 & 111 & 49 & 117 & 48 \\
\hline$R X$ & 18 & 5 & 19 & 14 \\
\hline R1 & 25 & 5 & 26 & 33 \\
\hline
\end{tabular}

${ }^{\dagger}$ mean value \pm standard deviation. ${ }^{\ddagger}$ measured in $\%$ immunoreactive tumor cells for Ki-67. G1: well-differentiated; G2: moderately differentiated; G3: poorly differentiated; G4: undifferentiated. L0: No lymphangio invasion. L1: lymphangio invasion. V0: no vascular invasion; V1: vascular invasion. Pn0: no perineural invasion; Pn1: perineural invasion. R0: no residual tumor; R1: microscopic residual tumor; RX: residual tumor unknown.

Immunohistochemistry (IHC) was performed on formalin-fixed, paraffin-embedded sections, cut at maximum $4 \mu \mathrm{m}$ thickness. Syndecan-1 expression was detected using a mouse anti-human monoclonal antibody (CD138 Clone MI15, Dako, Germany) in a dilution of 1:1000, according to the manufacturers' recommendations. IHC stains were manually evaluated on each TMA core by scoring intensity between 0 and 3 (Figure 1) [14]. To improve accuracy, we scored in 0.5 increments. Complete circumferential membrane staining was defined to be at least a score of 1.5 , the actual score depending on intensity. Manual scoring aimed for qualitative assessment of tumor cells, counting even single tumor cells. For patients with more than one TMA core of the same tumor taken for internal assessment of tumor heterogeneity, we calculated the mean value. 


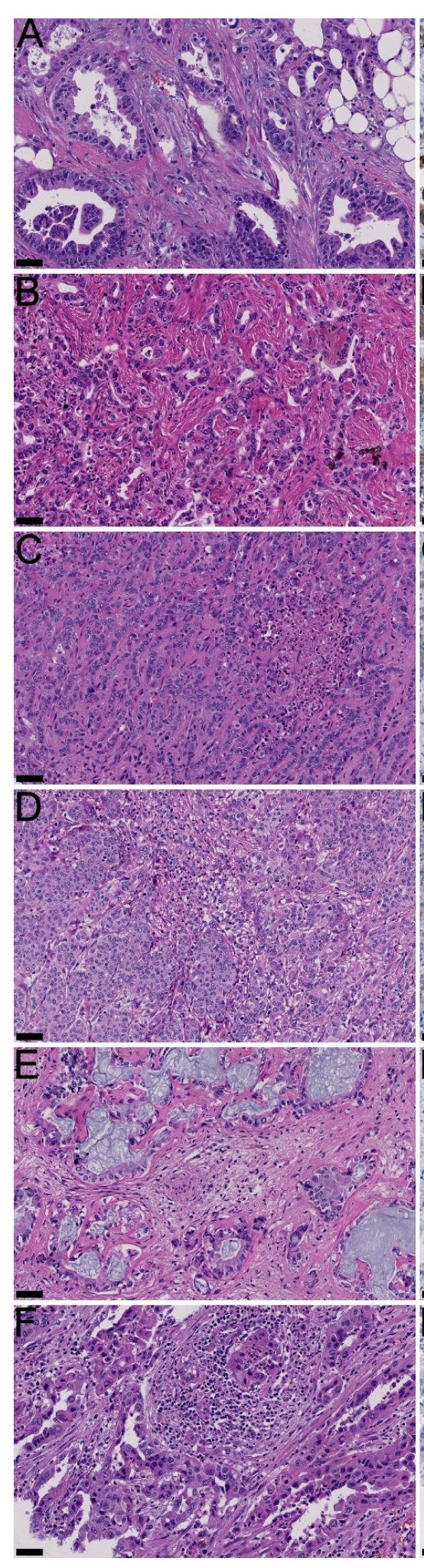

(HE)

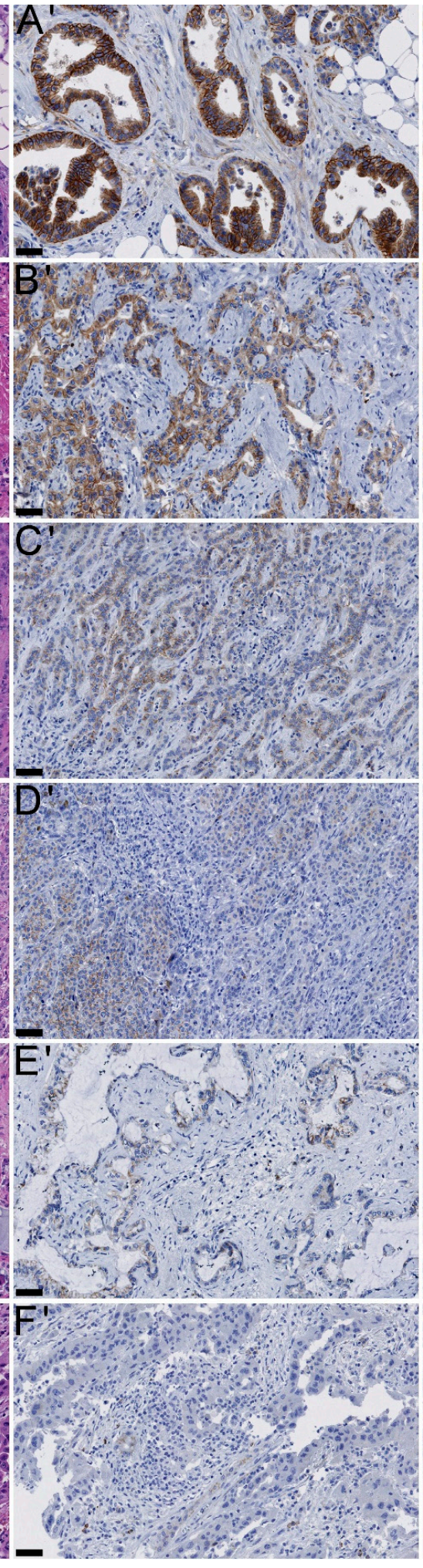

(Syndecan-1 IHC)

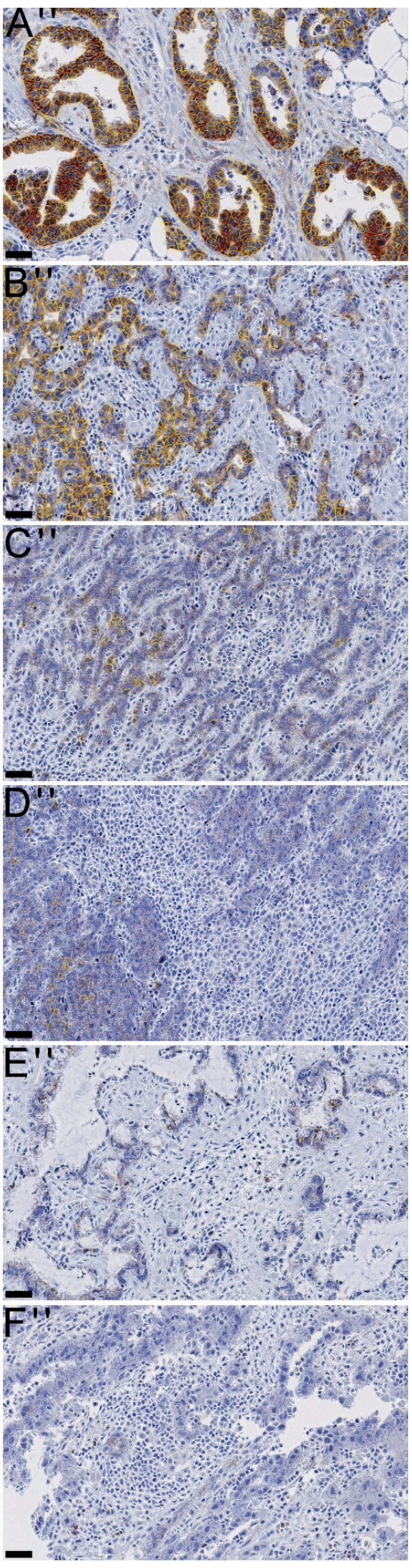

(QuPath)

Figure 1. Scoring of syndecan-1 immunohistochemistry: Manual score: Score $3.0\left(\mathbf{A}^{\prime}\right), 2.5\left(\mathbf{B}^{\prime}\right), 2.0\left(\mathbf{C}^{\prime}\right), 1.5\left(\mathbf{D}^{\prime}\right), 1.0\left(\mathbf{E}^{\prime}\right), 0.5$ $\left(\mathbf{F}^{\prime}\right)$. QuPath H-score $90.00\left(\mathbf{A}^{\prime \prime}\right), 52.62\left(\mathbf{B}^{\prime \prime}\right), 1.84\left(\mathbf{C}^{\prime \prime}\right), 1.02\left(\mathbf{D}^{\prime \prime}\right), 0.99\left(\mathbf{E}^{\prime \prime}\right), 0.05\left(\mathbf{F}^{\prime \prime}\right)$. Intrahepatic cholangiocarcinoma of small $(\mathbf{C}, \mathbf{D})$ and large $(\mathbf{A}, \mathbf{B}, \mathbf{E}, \mathbf{F})$ duct type are depicted. Classification of tumor staining intensity: red circumference $3+$, orange $2+$, yellow $1+$, blue 0 ; stroma in white $\left(\mathbf{A}^{\prime \prime}-\mathbf{F}^{\prime \prime}\right)$. Black bars: each $50 \mu \mathrm{m}$. 
Quantitative assessment of syndecan-1 expression levels was performed using QuPath, an open-source bioimage analysis software, version 0.2.3 [15]. TMAs cores were dearrayed. Stain vectors and background vectors were individually set in each slide and further analyzed using the "cell detection" algorithm in QuPath. Cellular chromogen 3,3'-diaminobenzidine-tetrahydrochloride-dihydrate mean levels were classified in four categories using empirical threshold scores $(0-0.21$ for score $0,>0.21$ for $1+,>0.45$ for $2+$, and $>0.7$ for $3+$; see Figure 1). These levels provided a relatively low threshold for the discrimination of negative stains (score 0 ) and low positivity (score $1+$ ), and a relatively high threshold for the highest achievable score (score 3+). Tumor cells were annotated using a detection classifier. Due to intertumoral heterogeneity, we used custom-tailored classifiers on a case-to-case basis to ensure proper separation of tumor and non-tumor tissue. This entailed the application of the random trees classifier to train QuPath interactively to distinguish tumor cells from stromal cells. The H-score was calculated according to established practice. It is calculated from the extent and intensity of staining, giving a score range of 0 to 300 [16]. In addition, to further elucidate the relationship between syndecan-1, $K R A S$, and patient prognosis, we analyzed the publicly available cBioPortal for Cancer Genomics data for cholangiocarcinoma data from the Cancer Genome Atlas (TCGA) and non TCGA data [17-19].

Using the syndecan-1 H-scores from our cohort and the SDC1 mRNA data from the TCGA cohort, both optimal cut-off values were calculated using the Charité Cutoff Finder [20]. Survival analyses were plotted by the Kaplan-Meier model and compared by the log-rank test. For the survival analysis of our cohort, we used only primary iCCA, and we excluded cases with irresectability and recurrent tumors. The database patient/TMAcore assignment was conducted using the MS-Access 2016 to achieve referential integrity. We calculated the Spearman rank-order correlation coefficient for ordinal data and Pearson correlation coefficient for metric data using SPSS v27.0.1.0 (two-sided). To compare the means of two groups, we used Student's $t$-test. A $p$-value $<0.05$ was considered statistically significant.

\section{Results}

\subsection{Syndecan-1 Expression Was Downregulated during Cholangiocarcinogenesis}

To investigate syndecan-1 in cholangiocarcinoma, we performed immunohistochemical analyses using our large cohort of over 470 iCCA, PHCC, dCCA, and GBC. Syndecan-1 was uniformly expressed cytoplasmically and at the cell membranes of hepatocytes and occasionally of bile ducts, as well as in singular plasma cells in normal liver. According to the literature, carcinomas have been attributed a "honeycomb" staining pattern as well heterogenous staining intensity of cell membranes and cytoplasm [4,21,22]. At least weak (intensity score $\geq 0.5$ ) syndecan- 1 expression was detected in $81.42 \%$ of iCCA, $62.39 \%$ of PHCC, $41.18 \%$ of eCCA, $44.86 \%$ of GBC, $85.94 \%$ of Billn, $76.19 \%$ of small bile ducts, $78.38 \%$ of large and perihilar bile ducts, and $28.57 \%$ of gallbladder epithelium. To our knowledge, only one study has analyzed immunohistochemical syndecan-1 expression in cholangiocarcinoma, revealing a positivity in 39.1\%, without subclassification [5]. Overall, Billn $(n=155)$ showed a comparable mean syndecan- 1 H-score $(94.36 \pm 65.42)$ to large and perihilar bile ducts $(n=91 ; 91.46 \pm 67.53),(p=0.741)$. The mean H-score of iCCA, dCCA, PHCC, and GBC had a significantly lower mean syndecan-1 H-score than normal bile ducts $(p<0.001)$, arguing towards a significant downregulation of syndecan-1 during cholangiocarcinogenesis (see Figure 2$)$. Lymph node metastases $(n=126 ; 52.53 \pm 50.11)$, however, showed a significantly higher mean value than primary carcinomas $(p=0.034)$. The mean intensity score in normal bile ducts was $1.63 \pm 0.86$, in Billn $2.2 \pm 0.73$, in lymph node metastasis $1.61 \pm 0.88$, while primary carcinomas showed a mean score of $1.41 \pm 0.81$. On the contrary, according to Yao et al. (2020), in PDAC and pancreatic intraepithelial neoplasia, immunohistochemistry showed a higher syndecan-1 intensity than in normal tissue [7]. 

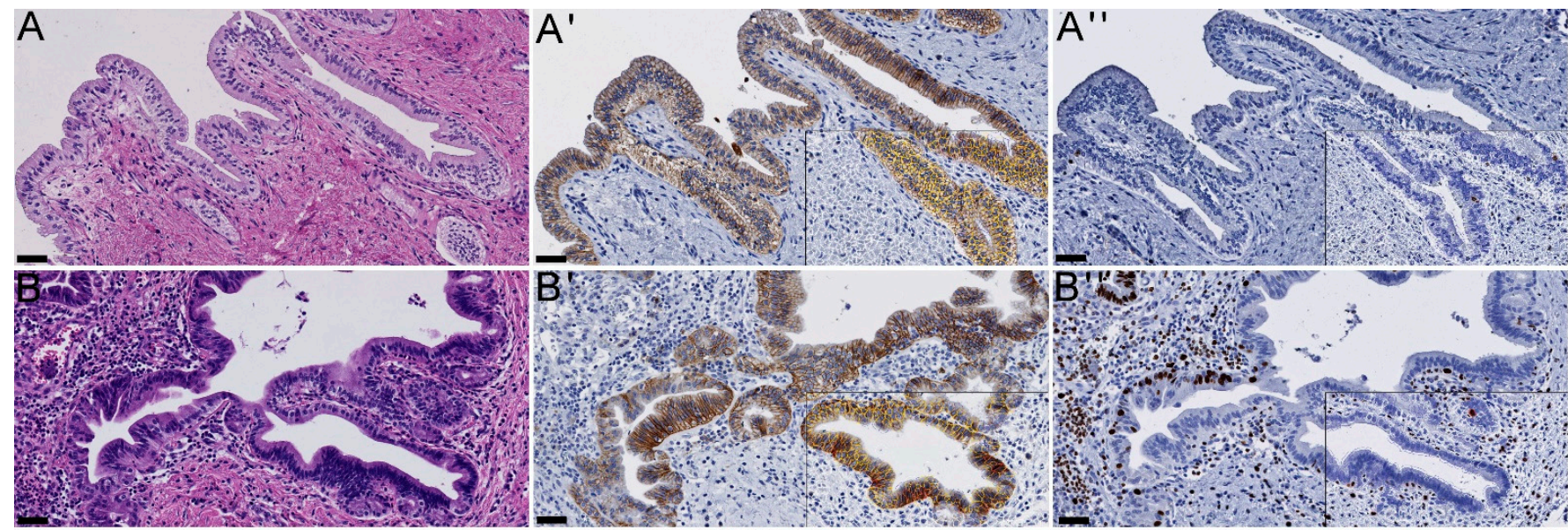

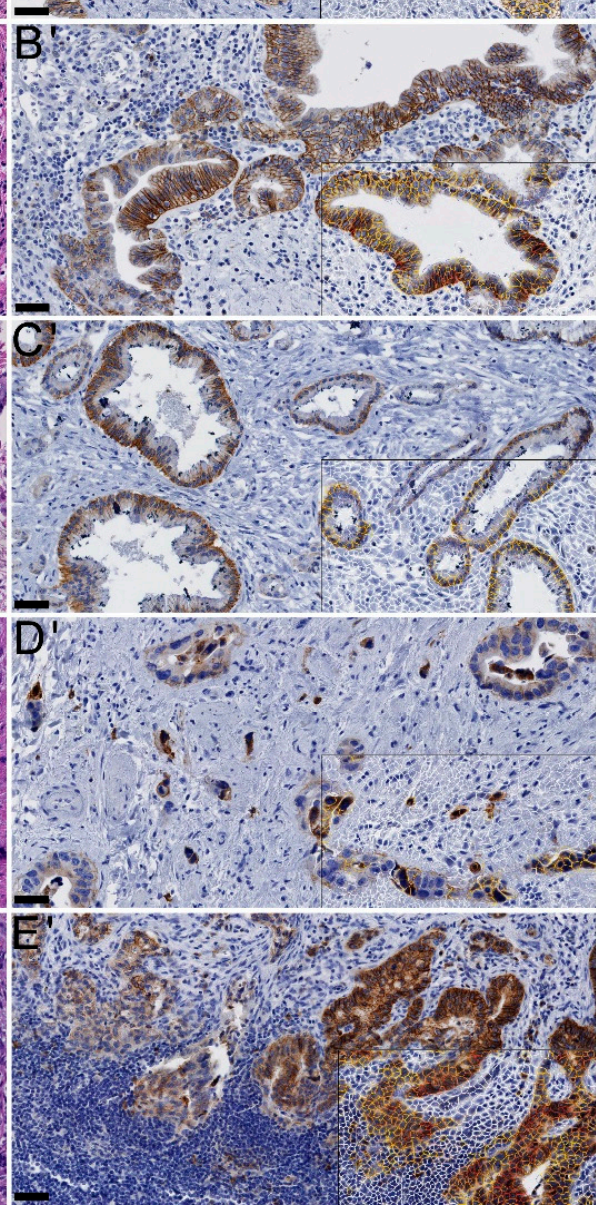

(Syndecan-1 IHC)

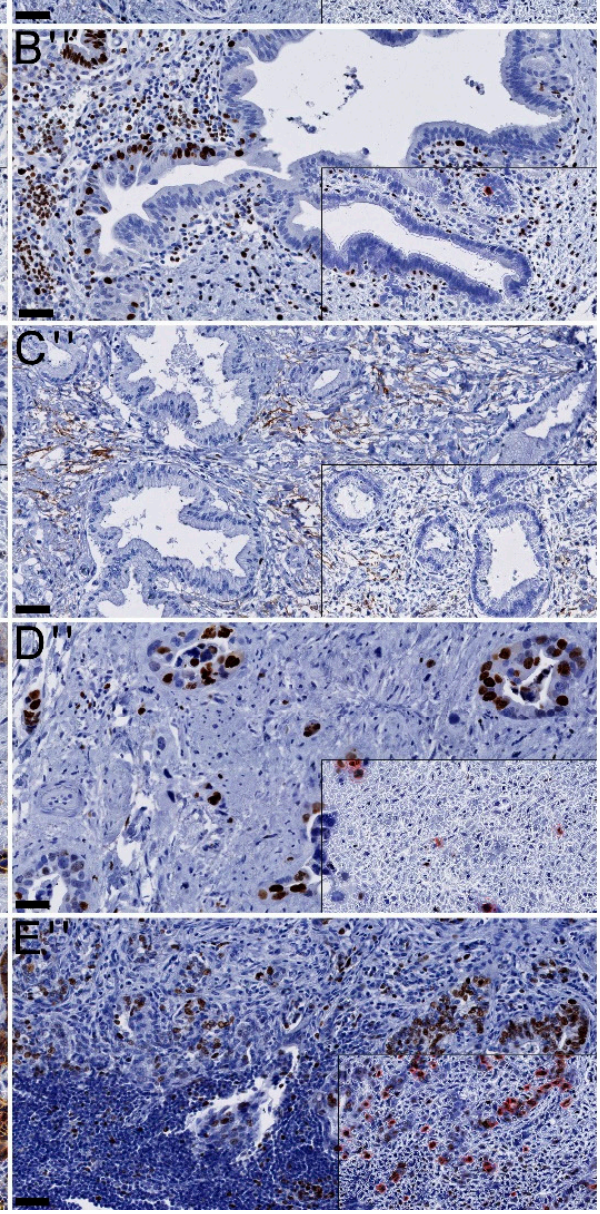

(Ki-67 IHC)

Figure 2. Syndecan-1 expression decreased in cholangiocarcinoma: In normal bile ducts (A), biliary intraepithelial neoplasia (B), and lymph node metastases (E), syndecan-1 staining was higher than in well- (C) and poorly differentiated (D) cholangiocarcinoma. (A) Perihilar bile duct, (B) low-grade BilIn, (C-E) PHCC. Syndecan-1 H-score: 79.81 (A), 81.22 (B), 45.20 (C), 34.70 (D), 106.31 (E). Ki-67: 0.06\% (A), 9.07\% (B), 0.98\% (C), 41.67 (D), 32.51 (E). The overlay depicting QuPathanalysis is shown each on the bottom right $\left(\mathbf{A}^{\prime}-\mathbf{E}^{\prime}, \mathbf{A}^{\prime \prime}-\mathbf{E}^{\prime \prime}\right)$. Classification of tumor staining intensity: red circumference $3+$, orange 2+, yellow $1+$, blue 0 ; stroma in white $\left(\mathbf{A}^{\prime}-\mathbf{E}^{\prime}\right)$; red circumference positive, blue negative; stroma in white ( $\left.\mathbf{A}^{\prime \prime}-\mathbf{E}^{\prime \prime}\right)$.

Black bars: each $50 \mu \mathrm{m}$.

\subsection{Low Syndecan-1 mRNA Levels Were Associated with Better Survival}

To comprehensively investigate syndecan-1 concerning patient prognosis, we conducted survival analysis (Figure 3). In our cohort, 122 patients met the inclusion criteria (see Table 1). Low mRNA levels of syndecan-1 (gene symbol SDC1) were significantly associated with a better prognosis (cutoff value 6962, area under the curve (AUC) =0.64; see Figure $3 a$ ). In the TCGA cohort, two patients showed a missense mutation of KRAS. Both were in the high SDC1 mRNA group. Immunohistochemical expression of syndecan1 , as evaluated by H-scores, could not separate any prognostically relevant groups (see Figure $3 b$ ). The best cutoff value in our cohort was the H-score of 28.01 (AUC $=0.55$ ). 


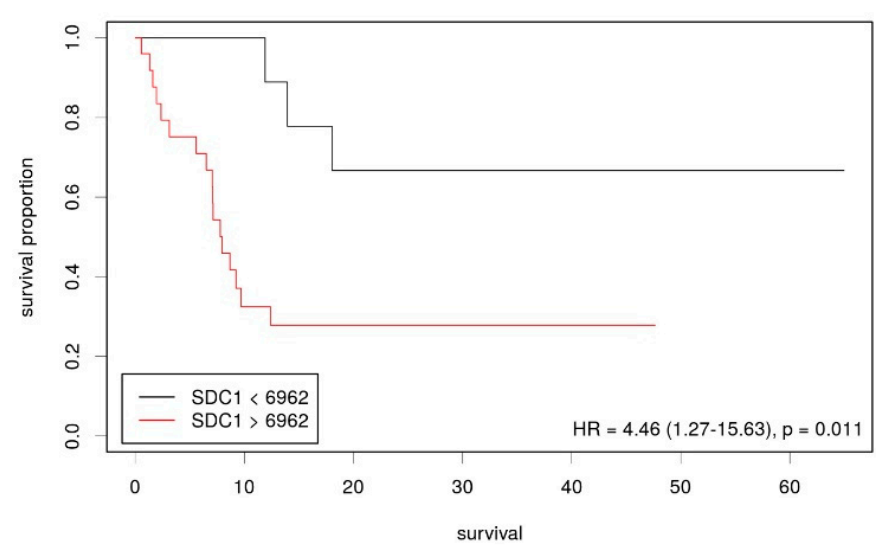

(a)

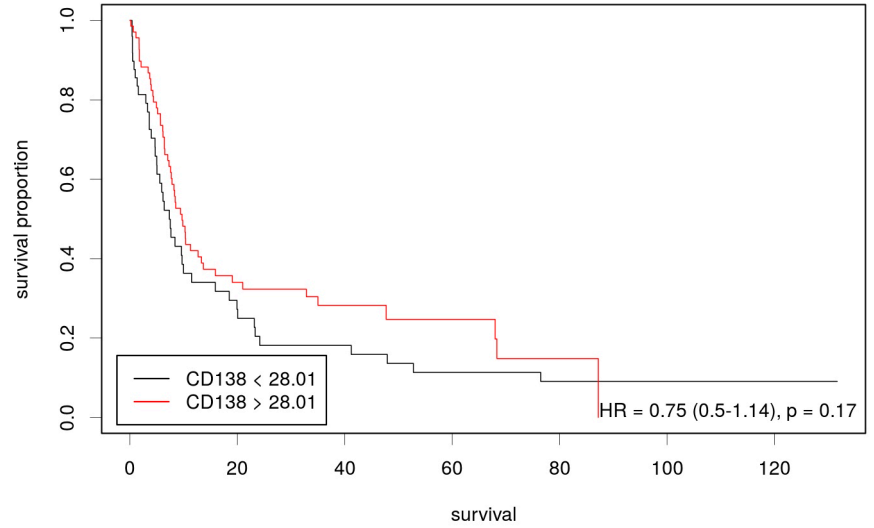

(b)

Figure 3. Kaplan-Meier curves for disease-free survival of cholangiocarcinoma (a) and recurrence-free survival of intrahepatic cholangiocarcinoma (b). Patients stratified by (a) SDC1 mRNA expression (TCGA cohort; $n=36$ ) and (b) syndecan-1 immunohistochemical expression level (own cohort; $n=122)$. $x$-axis: time in months $(\mathbf{a}, \mathbf{b})$.

Additionally, in iCCA, we performed a correlation analysis between the syndecan-1 $\mathrm{H}$-score and the residual tumor classification, the lymph node status, the iCCA subclassification (small and large duct type), and the Ki-67 proliferation rate. As expected, the proliferation rate correlated with histological tumor grade [23]. No statistically significant association between the H-score and the prognostically important marker proliferation rate, residual tumor status, lymph node status, and histological subtype analysis was detected (Ki-67: $\rho=0.08(p=0.925)$, R-status: $\rho=0.062(p=0.484)$, iCCA subclassification $\rho=0.002$ $(p=0.985)$. In primary carcinomas, syndecan- 1 did not correlate with tumor size $\rho=0.14$ $(p=0.835)$, grading $\rho=0.063(p=0.187)$, or GPT-value $(\rho=0.49(p=0.714))$. However, syndecan-1 correlated weakly with age $\rho=0.138(p=0.003)$.

\subsection{Molecular Analysis}

In the overall cBioPortal data, KRAS was mutated in 122 of 805 cholangiocarcinoma cases (15.2\%; not shown). Most KRAS mutations were G12D (45), G12V (39), and G12C (7). In the TCGA cohort, two KRAS mutations were detected. SDC1 mRNA levels were not overtly associated with KRAS mutational status (Figure S1). In all cBio Portal cholangiocarcinomas with known progression-free survival, KRAS mutations were associated with a poor prognosis $(p<0.001$; see Figure S2).

\section{Discussion}

This is the first study to comprehensively study syndecan- 1 in cholangiocarcinoma to unravel its possible role as a therapeutic agent, as already shown for PDAC. We could demonstrate positivity in most iCCA, dCCA, GBC, PHCC, Billn, and associated normal tissue. In our study cohort of iCCA, the immunohistochemical expression did not correlate with recurrence-free survival. Only one other study, conducted by Harada and coauthors (2003), had previously addressed the question as to whether syndecan-1 is associated with patient prognosis in cholangiocarcinoma. This study demonstrated an association between reduced immunohistochemical expression of syndecan- 1 and reduced recurrence-free survival [4]. However, this study only included 33 cases, of which $13(39 \%)$ were poorly differentiated. Poor differentiation significantly correlated with syndecan-1 expression. We assume this served as a confounder, explaining the different results. In addition, the concept of subclassification of large and small duct type cholangiocarcinomas arose more than a decade after the aforementioned study, which may have impacted the different prognosis as well $[13,24]$.

mRNA levels could differentiate two different prognostically significant groups in the TCGA cohort. However, this was a small group $(n=36)$ compared to our large cohort 
( $n=122)$. mRNA levels may only be comparable to a limited extent to protein levels, as the extent to which the mRNA is translated to a protein may vary. In addition, high SDC1 mRNA levels in whole tissue lysates may originate from tumor-infiltrating plasma cells and not only carcinoma cells. Immunohistochemistry, on the other hand, is direct, though semiquantitative measurement of the protein of interest and evaluation concentrates on tumor cells only. Therefore, immunohistochemistry is widely used in routine pathology as a derivate marker, e.g., for the analysis of Her2 expression in breast cancer.

Syndecan-1 is a membrane-bound protein. However, regulated nuclear translocation has been described via association with tubulin in the mitotic spindle [25]. These findings suggest an association with cell proliferation and tumor aggressiveness. However, in our study, there was no positive correlation with the Ki-67 proliferation index.

Elevated syndecan-1 expression had been demonstrated in KRAS-driven PDAC [7]. In our series, syndecan-1 staining intensity was significantly lower in cholangiocarcinoma when compared to normal bile ducts. There was no significant difference between normal bile ducts and biliary intraepithelial neoplasia. In intrahepatic cholangiocarcinoma, syndecan-1 H-score had no significant influence on recurrence-free survival, although KRAS-mutation status had an influence on recurrence-free survival in cholangiocarcinoma of the cBioPortal cholangiocarcinoma cohort. This discrepancy in syndecan-1 immunohistochemistry may have multiple reasons. First and foremost, KRAS mutations are much less common in cholangiocarcinoma (15.2\%) when compared to PDAC (more than $90 \%$ ). Due to KRAS-dependent changes, syndecan-1 is upregulated in PDAC, as stated by Yao et al. [7]. For this reason, to reliably compare cholangiocarcinoma and PDAC, two subgroups may be needed to further depict an independent role of syndecan-1, namely, KRAS-mutated and KRAS-wildtype tumors. Second, PDAC and cholangiocarcinoma are embryonal related but show distinct tumor biology. This may result in different tumor evolution and cell signaling. In comparison to our molecular analysis, Robertson et al. found $7.4 \%$ KRAS mutations (G12D) in a cohort of 54 intrahepatic cholangiocarcinomas, which were associated with a worse long-term overall survival [26]. In cholangiocarcinoma, the frequency of KRAS mutations is dependent on the subtype. In a recent study, Goeppert et al. demonstrated $23.3 \%$ KRAS alterations in iCCA, $40.6 \%$ in PHCC, $16.7 \%$ in dCCA, and $24.4 \%$ in GBC. Patients with tumors harboring KRAS mutations showed a significantly poorer survival [27]. These concordant findings underline the importance of further studies regarding the role of syndecan-1 in cholangiocarcinoma.

Our study has several limitations. First, syndecan-1 expression on tumor cells using immunohistochemistry is on a continuous spectrum, whereas in our evaluation, categorical and metrical variables need to be defined. To compensate for this, we manually scored each TMA core, counting even single cells, and, additionally, for quantification, we calculated the $\mathrm{H}$-score. Second, in this study, we did not perform molecular analyses to determine whether SDC1 mRNA levels may correlate with immunohistochemical expression. Third, we did not compare different chemotherapy approaches for intrahepatic cholangiocarcinoma with syndecan-1 expression, although there may be an association. However, in our study using predominantly operation specimens of patients undergoing resection with curative intent, there had not been a sufficient number of patients that underwent prior chemotherapy treatment, especially considering the many subgroups needed for this analysis. Recent and future advances in therapies may increase the proportions of patients with neoadjuvant treatment $[12,28]$. Fourth, we investigated cholangiocarcinoma TMAs instead of whole slides. Although TMAs are a generally accepted method, naturally, immunohistochemistry of whole slides may have led to more accurate data. To compensate for this, in our series, we used more than 2100 2-mm measuring TMA cores, resulting in multiple scores per case.

To conclude, we could not show an impact of syndecan- 1 on patient prognosis in cholangiocarcinoma, at least none that could be shown immunohistochemically. It remains unclear as to whether SDC1 mRNA levels may reliably predict prognosis. Given the importance of syndecan-1 in PDAC, this is rather surprising and should warrant further investigation. Further experimental investigations are therefore needed to clarify whether 
syndecan-1 may predict chemotherapy response in cholangiocarcinoma and whether macropinocytosis may be involved in cholangiocarcinoma progression.

Supplementary Materials: The following are available online at https:/ / www.mdpi.com/article/10 $.3390 /$ jcm10132745/s1, Figure S1: TCGA mRNA analysis of cholangiocarcinoma, Figure S2: KRAS mutations in cholangiocarcinoma.

Author Contributions: Conceptualization, T.S.G., D.C.W. and B.K.S.; data curation, T.S.G., F.B., L.K.H. and H.L.; formal analysis, T.S.G., M.S. and B.K.S.; investigation, T.S.G.; methodology, T.S.G. and M.S.; project administration, B.K.S.; software, T.S.G.; supervision, W.R., H.L., and B.K.S.; validation, B.K.S.; visualization, T.S.G.; writing—original draft, T.S.G.; writing-review and editing, B.K.S. All authors have read and agreed to the published version of the manuscript.

Funding: This research received no external funding.

Institutional Review Board Statement: The study was conducted according to the guidelines of the Declaration of Helsinki and approved by the Institutional Ethics Committee (837.280.17 (11114) and 2021-15819).

Informed Consent Statement: The study has been conducted in full accordance with ethical principles, including the World Medical Association Declaration of Helsinki.

Data Availability Statement: Publicly available datasets were analyzed in this study. The results shown here are in part based upon data generated by the cBioPortal for Cancer Genomics data for cholangiocarcinoma. The data that support the findings of this study are available on request from the corresponding author. The data are not publicly available due to containing information that could compromise the privacy of research participants.

Acknowledgments: T.S.G. is supported by the Clinician Scientist Fellowship "Else Kröner Research College: 2018_Kolleg.05". Tissue samples were provided by the tissue bank of the University Medical Center Mainz in accordance with the regulations of the tissue bank. We thank Bonny Adami for excellent technical assistance.

Conflicts of Interest: The authors declare no conflict of interest.

\section{References}

1. Teng, Y.H.; Aquino, R.S.; Park, P.W. Molecular functions of syndecan-1 in disease. Matrix Biol. 2012, 31, 3-16. [CrossRef]

2. Regős, E.; Karászi, K.; Reszegi, A.; Kiss, A.; Schaff, Z.; Baghy, K.; Kovalszky, I. Syndecan-1 in Liver Diseases. Pathol. Oncol. Res. 2020, 26, 813-819. [CrossRef]

3. Roskams, T.; Rosenbaum, J.; De Vos, R.; David, G.; Desmet, V. Heparan sulfate proteoglycan expression in chronic cholestatic human liver diseases. Hepatology 1996, 24, 524-532. [CrossRef]

4. Harada, K.; Masuda, S.; Hirano, M.; Nakanuma, Y. Reduced expression of syndecan-1 correlates with histologic dedifferentiation, lymph node metastasis, and poor prognosis in intrahepatic cholangiocarcinoma. Hum. Pathol. 2003, 34, 857-863. [CrossRef]

5. Kind, S.; Merenkow, C.; Büscheck, F.; Möller, K.; Dum, D.; Chirico, V.; Luebke, A.M.; Höflmayer, D.; Hinsch, A.; Jacobsen, F.; et al. Prevalence of Syndecan-1 (CD138) Expression in Different Kinds of Human Tumors and Normal Tissues. Dis. Markers 2019, 2019, 4928315. [CrossRef] [PubMed]

6. Jayashankar, V.; Edinger, A.L. Macropinocytosis confers resistance to therapies targeting cancer anabolism. Nat. Commun. 2020, 11, 1121. [CrossRef]

7. Yao, W.; Rose, J.L.; Wang, W.; Seth, S.; Jiang, H.; Taguchi, A.; Liu, J.; Yan, L.; Kapoor, A.; Hou, P.; et al. Syndecan 1 is a critical mediator of macropinocytosis in pancreatic cancer. Nature 2019, 568, 410-414. [CrossRef]

8. Hobbs, G.A.; Baker, N.M.; Miermont, A.M.; Thurman, R.D.; Pierobon, M.; Tran, T.H.; Anderson, A.O.; Waters, A.M.; Diehl, J.N.; Papke, B.; et al. Atypical KRAS(G12R) Mutant Is Impaired in PI3K Signaling and Macropinocytosis in Pancreatic Cancer. Cancer Discov 2020, 10, 104-123. [CrossRef] [PubMed]

9. Liu, H.; Sun, M.; Liu, Z.; Kong, C.; Kong, W.; Ye, J.; Gong, J.; Huang, D.C.S.; Qian, F. KRAS-enhanced macropinocytosis and reduced FcRn-mediated recycling sensitize pancreatic cancer to albumin-conjugated drugs. J. Control Release 2019, $296,40-53$. [CrossRef]

10. Pupo, E.; Avanzato, D.; Middonti, E.; Bussolino, F.; Lanzetti, L. KRAS-Driven Metabolic Rewiring Reveals Novel Actionable Targets in Cancer. Front. Oncol. 2019, 9, 848. [CrossRef]

11. Banales, J.M.; Marin, J.J.G.; Lamarca, A.; Rodrigues, P.M.; Khan, S.A.; Roberts, L.R.; Cardinale, V.; Carpino, G.; Andersen, J.B.; Braconi, C.; et al. Cholangiocarcinoma 2020: The next horizon in mechanisms and management. Nat. Rev. Gastroenterol. Hepatol. 2020, 17, 557-588. [CrossRef] [PubMed] 
12. Akateh, C.; Ejaz, A.M.; Pawlik, T.M.; Cloyd, J.M. Neoadjuvant treatment strategies for intrahepatic cholangiocarcinoma. World J. Hepatol. 2020, 12, 693-708. [CrossRef] [PubMed]

13. Akita, M.; Fujikura, K.; Ajiki, T.; Fukumoto, T.; Otani, K.; Azuma, T.; Itoh, T.; Ku, Y.; Zen, Y. Dichotomy in intrahepatic cholangiocarcinomas based on histologic similarities to hilar cholangiocarcinomas. Mod. Pathol. 2017, 30, 986-997. [CrossRef] [PubMed]

14. Remmele, W.; Stegner, H.E. Recommendation for uniform definition of an immunoreactive score (IRS) for immunohistochemical estrogen receptor detection (ER-ICA) in breast cancer tissue. Pathologe 1987, 8, 138-140.

15. Bankhead, P.; Loughrey, M.B.; Fernández, J.A.; Dombrowski, Y.; McArt, D.G.; Dunne, P.D.; McQuaid, S.; Gray, R.T.; Murray, L.J.; Coleman, H.G.; et al. QuPath: Open source software for digital pathology image analysis. Sci. Rep. 2017, 7, 16878. [CrossRef]

16. McCarty, K.S., Jr.; Szabo, E.; Flowers, J.L.; Cox, E.B.; Leight, G.S.; Miller, L.; Konrath, J.; Soper, J.T.; Budwit, D.A.; Creasman, W.T.; et al. Use of a monoclonal anti-estrogen receptor antibody in the immunohistochemical evaluation of human tumors. Cancer Res. 1986, 46, 4244s-4248s.

17. Cerami, E.; Gao, J.; Dogrusoz, U.; Gross, B.E.; Sumer, S.O.; Aksoy, B.A.; Jacobsen, A.; Byrne, C.J.; Heuer, M.L.; Larsson, E.; et al. The cBio cancer genomics portal: An open platform for exploring multidimensional cancer genomics data. Cancer Discov. 2012, 2, 401-404. [CrossRef]

18. Gao, J.; Aksoy, B.A.; Dogrusoz, U.; Dresdner, G.; Gross, B.; Sumer, S.O.; Sun, Y.; Jacobsen, A.; Sinha, R.; Larsson, E.; et al. Integrative analysis of complex cancer genomics and clinical profiles using the cBioPortal. Sci. Signal. 2013, 6, pl1. [CrossRef]

19. Farshidfar, F.; Zheng, S.; Gingras, M.C.; Newton, Y.; Shih, J.; Robertson, A.G.; Hinoue, T.; Hoadley, K.A.; Gibb, E.A.; Roszik, J.; et al. Integrative Genomic Analysis of Cholangiocarcinoma Identifies Distinct IDH-Mutant Molecular Profiles. Cell Rep. 2017, 18, 2780-2794. [CrossRef]

20. Budczies, J.; Klauschen, F.; Sinn, B.V.; Győrffy, B.; Schmitt, W.D.; Darb-Esfahani, S.; Denkert, C. Cutoff Finder: A comprehensive and straightforward Web application enabling rapid biomarker cutoff optimization. PLoS ONE 2012, 7, e51862. [CrossRef]

21. Conejo, J.R.; Kleeff, J.; Koliopanos, A.; Matsuda, K.; Zhu, Z.W.; Goecke, H.; Bicheng, N.; Zimmermann, A.; Korc, M.; Friess, H.; et al. Syndecan-1 expression is up-regulated in pancreatic but not in other gastrointestinal cancers. Int. J. Cancer 2000, 88, 12-20. [CrossRef]

22. Roskams, T.; De Vos, R.; David, G.; Van Damme, B.; Desmet, V. Heparan sulphate proteoglycan expression in human primary liver tumours. J. Pathol. 1998, 185, 290-297. [CrossRef]

23. Aloysius, M.M.; Hewavisenthi, S.J.; Bates, T.E.; Rowlands, B.J.; Lobo, D.N.; Zaitoun, A.M. Predictive value of tumor proliferative indices in periampullary cancers: Ki-67, mitotic activity index (MI) and volume corrected mitotic index (M/V) using tissue microarrays. World J. Surg. 2010, 34, 2115-2121. [CrossRef]

24. Aishima, S.; Oda, Y. Pathogenesis and classification of intrahepatic cholangiocarcinoma: Different characters of perihilar large duct type versus peripheral small duct type. J. Hepatobiliary Pancreat. Sci. 2015, 22, 94-100. [CrossRef]

25. Brockstedt, U.; Dobra, K.; Nurminen, M.; Hjerpe, A. Immunoreactivity to cell surface syndecans in cytoplasm and nucleus: Tubulin-dependent rearrangements. Exp. Cell Res. 2002, 274, 235-245. [CrossRef]

26. Robertson, S.; Hyder, O.; Dodson, R.; Nayar, S.K.; Poling, J.; Beierl, K.; Eshleman, J.R.; Lin, M.T.; Pawlik, T.M.; Anders, R.A. The frequency of KRAS and BRAF mutations in intrahepatic cholangiocarcinomas and their correlation with clinical outcome. Hum. Pathol. 2013, 44, 2768-2773. [CrossRef]

27. Goeppert, B.; Folseraas, T.; Roessler, S.; Kloor, M.; Volckmar, A.L.; Endris, V.; Buchhalter, I.; Stenzinger, A.; Grzyb, K.; Grimsrud, M.M.; et al. Genomic Characterization of Cholangiocarcinoma in Primary Sclerosing Cholangitis Reveals Therapeutic Opportunities. Hepatology 2020, 72, 1253-1266. [CrossRef]

28. Marabelle, A.; Le, D.T.; Ascierto, P.A.; Di Giacomo, A.M.; De Jesus-Acosta, A.; Delord, J.P.; Geva, R.; Gottfried, M.; Penel, N.; Hansen, A.R.; et al. Efficacy of Pembrolizumab in Patients With Noncolorectal High Microsatellite Instability/Mismatch Repair-Deficient Cancer: Results From the Phase II KEYNOTE-158 Study. J. Clin. Oncol. 2020, 38, 1-10. [CrossRef] 